\title{
A fundamental problem
}

\author{
Some materials scientists think that the United Kingdom's decision to cut funding for 'big \\ physics' is benefiting them. Here's why it isn't.
}

When the UK's Science and Technology Facilities Council (STFC) released its five-year plan in December 2007, highenergy physicists let out a collective gasp of horror. The spending proposal promised deep cuts to their grants, and in addition, announced the nation's immediate withdrawal from the International Linear Collider - the next-generation particle accelerator on which the future of particle physics is staked. They weren't the only ones burned by the proposed budget. Astronomers were threatened with the loss of one of their largest facilities, and solar terrestrial physicists would also see deep cuts to their funding. According to the council, the reductions are needed to make up for an $£ 80$ million spending shortfall.

But although these fields languish, materials science and nanotechnology have emerged as something of winners in the funding process. The linear collider was eschewed in part to ensure the full funding of two large facilities: the Diamond synchrotron light source and the ISIS second target, both at Rutherford Appleton Laboratory in Oxfordshire. These two machines provide $\mathrm{X}$-rays and neutrons respectively, which can be used (among other things) as diagnostic probes of various materials. In addition, the Engineering and Physical Sciences Research Council (EPSRC), which funds the lion's share of materials research, is expected to see its $£ 740$ million budget rise by nearly $19 \%$ between now and 2010/11.

Privately at least, some materials researchers are pleased about the current state of affairs. 'Big toys' such as particle accelerators and telescopes gobble up billions in funding, and they believe that money might now be redirected towards their table-top basic science.

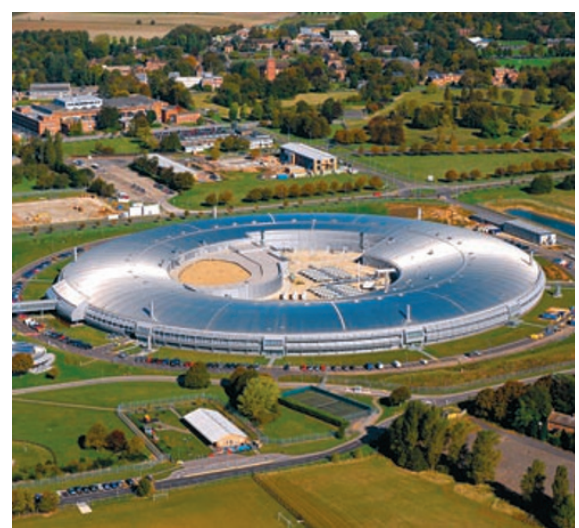

to cut investigator-led grants by $15 \%$ over three years in order to fund more top-down initiatives. Meanwhile, under a reorganization announced in April of this year, EPSRC has consolidated its physics and chemistry programmes into a single "physical sciences" panel; materials science is wedged into a larger programme that includes mechanical and medical engineering. All this is done with the stated goal to "maximise the economic and social impact of the research and training we fund". In other words, basic, investigator-driven science is taking a backseat to more multidisciplinary and applied projects.

Taken in tandem, the changes show

The Diamond light source is being funded at the expense of fields such as particle physics.

But the UK government has other plans. In past years, the government has become increasingly insistent that science provide the country with fiscal gain. A 2004 blueprint for science (commissioned by Gordon Brown, who was then chancellor) called for tying government-backed research "to tangible economic benefits". And a 2006-2007 review of all the UK's research councils recommended that "economic impact" be included for the first time in peer-review.

At STFC the result of this policy is clear. Fields such as high-energy physics and astronomy face cuts because they struggle to show how they provide pay-off to the rest of society. Meanwhile, more 'practical' facilities such as Diamond and ISIS will be funded at full cost.

At EPSRC, where funding is still on the rise, a more subtle effect can be seen. Despite increases, the council plans that, in the case of physics at least, the British government fails to understand how science is done. In some cases, such as sustainable energy, information and biotechnologies, funding research into new materials applications and devices can indeed provide economic benefit. But a systematic emphasis on applied research will yield little. The benefits of scientific progress come not from financial gain but from long-term fundamental research, education and surreptitious discovery.

There is certainly room for debate over whether materials science or highenergy physics is more deserving of funding. But that debate should be based on scientific rather than economic merits. For now, materials scientists should stand with the injured fields and convince the British government that science should not be subsumed by industrial needs. All scientists have a duty to support their colleagues from high-energy physics and astronomy and demand that the funding councils renew their commitment to fundamental research. 